Article

\title{
Positive and Negative Affect More Concurrent among Blacks than Whites
}

\author{
Maryam Moghani Lankarani ${ }^{1,2}$ and Shervin Assari 1,3,* \\ 1 Department of Psychiatry, University of Michigan, Ann Arbor 4250 Plymouth Road, SPC 5763, Ann Arbor, \\ MI 48109-2700, USA; lankaranii@yahoo.com \\ 2 Medicine and Health Promotion Institute, Mollasadra Avenue, Tehran 1991, Iran \\ 3 Center for Research on Ethnicity, Culture and Health, School of Public Health, University of Michigan, \\ Ann Arbor, MI 48109-2700, USA \\ * Correspondence: assari@umich.edu; Tel.: +1-734-232-0445; Fax: +1-734-615-8739
}

Received: 23 June 2017; Accepted: 18 July 2017; Published: 1 August 2017

\begin{abstract}
Background: While positive and negative affect are inversely linked, people may experience and report both positive and negative emotions simultaneously. However, it is unknown if race alters the magnitude of the association between positive and negative affect. The current study compared Black and White Americans for the association between positive and negative affect. Methods: We used data from MIDUS (Midlife in the United States), a national study of Americans with an age range of 25 to 75. A total number of 7108 individuals were followed for 10 years from 1995 to 2004. Positive and negative affect was measured at baseline (1995) and follow-up (2004). Demographic (age and gender), socioeconomic (education and income) as well as health (self-rated health, chronic medical conditions, and body mass index) factors measured at baseline were covariates. A series of linear regressions were used to test the moderating effect of race on the reciprocal association between positive and negative affect at baseline and over time, net of covariates. Results: In the pooled sample, positive and negative affect showed inverse correlation at baseline and over time, net of covariates. Blacks and Whites differed in the magnitude of the association between positive and negative affect, with weaker inverse associations among Blacks compared to Whites, beyond all covariates. Conclusion: Weaker reciprocal association between positive and negative affect in Blacks compared to Whites has implications for cross-racial measurement of affect and mood, including depression. Depression screening programs should be aware that race alters the concordance between positive and negative affect domains and that Blacks endorse higher levels of positive affect compared to Whites in the presence of high negative affect.
\end{abstract}

Keywords: positive affect; negative affect; ethnic groups; Blacks; African Americans; Whites

\section{Introduction}

In 1969, Norman Bradburn showed that positive and negative affect are two separate, but inter-connected emotions with moderate negative associations [1,2]. Positive affect and emotions include happiness, joy, contentment, interest and love [3-7]; negative affect and emotions include sadness, guilt, fear, anger, and disgust [8-10]. While positive affect promotes health [11] and reduces risk of mortality [12] via better psychological adjustment [13], negative affect increases risk of chronic disease [14] as well as mortality [15] via vigilance, threat [16], and an unhealthy lifestyle [17].

Positive affect, a predictor of creation [18] and openness [19], is linked to lower autonomic reactivity [20] and better physical and mental health [21]. Constant high levels of negative affect, however, predisposes individuals to emotional and health problems [8-10]. The overall role of negative affect as a common risk factor for a wide range of emotional and psychiatric disorders, such 
as anxiety and depression, is well established, and may partially explain why several disorders tend to co-occur [22].

In addition to their physical health effects [23], positive and negative affect have major implications for diagnosis of mood disorders, particularly depression [24]. Positive and negative emotions compose major factors in mood disorders such as depression [25-27] and bipolar disorder [28]. As racial groups differ in their tendency to experience or express positive and negative affect $[29,30]$, there is a need for cross-racial studies that compare racial groups for association between positive and negative affect [31-38].

Whether or not race alters simultaneous experience and presentation of positive and negative affect is still unknown $[29,30]$. While some studies have suggested that the factorial structure of depression scales is invariant across racial groups [39-41], other studies have shown that the degree by which positive and negative affect correlate may depend on race and ethnicity $[29,42,43]$. While there is an ongoing debate whether or not positive and negative affect similarly represent depression among Whites and Blacks [38-41,44-50], there are studies showing higher reliability of negative affect despite lower reliability of depression measures for Blacks compared to Whites [40,41,44]. Thus, positive and negative affect domains may differently compose the presentation of depression for Blacks and Whites [39].

In a recent study by Moazen-Zadeh and Assari, the item loading for the item "I was happy" to the Center for Epidemiologic Studies Depression Scale (CES-D) measure was 0.68 and -0.66 respectively for Blacks and Whites [29]. In another study, by Assari et al., negative affect showed stronger correlations with the Composite International Diagnostic Interview (CIDI)-based diagnosis of clinical depression in Blacks compared to Whites, while positive affect was similarly linked to MDD across race groups [45]. A national study found considerable variation in item loadings of the CES-D scale between Blacks and Whites. While negative affect items showed a better loading for Blacks, the positive affect items better loaded for Whites [5]. Canady et al. observed that the positive item "was happy" had different loadings on a depression scale between Blacks and Whites [43]. All these studies suggest that positive and negative affect may differently correlate between Blacks and Whites. These findings may help us better understand why correlates of depression and affect vary for Whites and Blacks [51-53], and why Blacks, who report higher levels of depressive symptoms (negative affect), do not endorse DSM criteria for the clinical disorder $[29,45,54]$.

While positive and negative affect are main factors in several depression scales [41,49,50,55], different populations may differ in how they experience or express positive and negative affect simultaneously [41,55]. Considering the gap in the literature on racial and ethnic variation in the concordance between positive and negative affect endorsement [29,41-43,45-48], more research is needed on moderating effects of race and ethnicity on the reciprocal links between positive and negative affect $[41,56,57]$.

We conducted the current study on racial differences in the bidirectional links between positive and negative affect, using a national sample of adults in the United States.

\section{Methods}

\subsection{Design}

This longitudinal study used data from MIDUS (http:/ / midus.wisc.edu), a 10 year longitudinal study conducted between 1995 and 2004. The study was a national cohort study of over 7000 American adults (aged 25 to 74). The study was carried out by the MacArthur Midlife Research Network. The main purpose of the study was to understand the role of psychosocial factors in age related variation in physical and mental health over the life course [58-62].

\subsection{Data Collection}

The survey used a multimodal data collection strategy, which was composed of a computer-assisted personal interview (CAPI), a computer-assisted telephone interview (CATI), a mail questionnaire, a telephone interview, and a face-to-face interview. First, the study employed an 
initial 30-minute phone interview followed by a set (two) of self-administered questionnaires (SAQs). SAQs were mailed to individuals who completed the phone interview [58-62].

\subsection{Ethics}

The study protocol was approved by the University of Michigan Institutional Review Board (IRB), and written informed consent was obtained for all participants. The study was funded by the National Institute on Aging (NIA). Monetary incentives were given at both Wave 1 and Wave 2 for compensation (US \$20 for completion of MIDUS 1 surveys and up to US \$60 for completion of MIDUS 2 surveys).

\subsection{Participants and Sampling}

The study used random digit dialing (RDD), which is a method for selecting people for involvement in telephone statistical surveys by generating telephone numbers at random in order to enroll a random sample of adults. The national RDD survey used telephone numbers within the continental United States as the sampling frame. The study used an oversampling in five cities (related to geographic-specific agendas), resulting in a baseline RDD sample of 4244 individuals. The sibling sample was then generated by a random selection of 529 cases from the RDD sample that had at least one sibling. Limited to siblings within a family that had the same biological mother and father, the study collected data from 950 siblings. The study also enrolled a twin sample, which used a two-part sampling design. The first part involved screening a representative national sample of approximately 50,000 households for the presence of a twin (as part of ongoing national omnibus surveys). The second part involved contacting the twin households and attempting to recruit twins (also aged 25-74) to participate in the survey. Cooperating twins were asked to provide contact information for their co-twin. The twin sample was ultimately composed of 957 twin pairs $(n=1914)$ [58-62]. As 1187 individuals were neither Whites nor Blacks, this study only included 5921 White and Black individuals.

Data collection of wave 1 was conducted in 1995 and 1996. The follow-up data collection was conducted in 2004 and 2005. Advance letters with an accompanying brochure were sent to all Time-1 participants, to remind them about their past participation and to inform them that an interviewer will contact them for the initial telephone survey in near future. After a phone interview, which lasted $30 \mathrm{~min}$ on average, participants received two SAQs via mail [58-62].

\subsection{Follow-Up Data}

From the total 7108 participants who were enrolled at baseline (completing the phone survey at MIDUS 1), data were gathered for 4963 (70\%) at MIDUS 2 9-10 years later. Overall retention rate was $75 \%$ in the MIDUS (adjusted for mortality). Major causes for non-participation at MIDUS 2 included refusal $(12 \%)$, could not be contacted $(10 \%)$, too ill to be interviewed $(8 \%)$, or deceased [58-62].

\subsection{Measures}

Predictor variables were those prominent in published research on survey participation and retention, including 7 core demographic variables and 10 physical health variables from either the baseline telephone interview or the SAQ.

\subsection{Demographic Characteristics}

Demographic variables were collected at baseline in 1995 and included age (continuous), gender $(0=$ male (reference group), $1=$ female), and race $(0=$ Whites (reference group $), 1=$ Blacks $)$.

Socioeconomic status. Socio-economic variables included educational level $(1=$ less than high school, 2 = high school graduate or equivalent, $3=$ some college, $4=$ college graduate or more) and personal income. Both variables were operationalized as continuous measures.

Physical Health. The following physical health variables were included in the study: self-rated health (SRH) $(1=$ worst, 10 = best), number of chronic medical conditions $(\mathrm{CMC})$, and body mass 
index (BMI). All measures were treated as continuous measures. While a higher score indicated better $\mathrm{SRH}$, higher scores for CMC and BMI reflected worse health.

Positive affect. Using a scale developed by Mroczek and Kolarz (1998), [63] positive affect was assessed using six items referring to the question: "During the past 30 days, how much of the time did you feel ... "Items included "cheerful", "in good spirits", "extremely happy", "calm and peaceful", "satisfied", and "full of life". Responses ranged from 1 (all of the time) to 5 (none of the time) for each item [63]. Mean positive affect scores were computed if at least one of the affect items were completed. Greater scores reflected more positive affect, with possible scores ranging from 1 to 5 . Internal consistency (reliability) was very good ( $\alpha=0.91$ for all, 0.91 for Whites, 0.92 for Blacks). Other studies have used this scale [64-66].

Negative affect. Using a scale developed by Mroczek and Kolarz (1998), [63] negative affect was assessed in response to six items referring to the question: "During the past 30 days, how much of the time did you feel ... " Negative affect items included "so sad", "nervous", "restless or fidgety", "hopeless", "worthless", and "everything was an effort". Responses ranged from 1 (all of the time) to 5 (none of the time) [63]. Mean negative affect scores were computed if at least one of the affect items were completed. Greater scores reflected more negative affect, with possible scores ranging from 1 to 5 . Internal consistency (reliability) was excellent ( $\alpha=0.86$ for all, 0.86 for Whites, 0.87 for Blacks). Other studies have used this scale [64-66].

\subsection{Statistical Note}

We used SPSS 20.0 for Windows (IBM Inc. Armonk, NY, USA) for data analysis. For univariate analysis we reported frequencies, percentages, and mean (standard deviations) in the pooled sample, as well as based on race. For bivariate associations, we used independent sample $t$ test, chi square test, as well as Pearson correlation test. For multivariable analysis, we ran a series of linear regressions in the pooled sample, with positive and negative affect as independent and dependent variables, respectively. In our first models, only demographics and socio-economics were controlled for. Subsequently, we controlled for health factors. First, we ran models without the interaction term. Then, we added the race by affect interaction term. We ran models with wave 1 and wave 2 positive and negative affect as outcomes. In the next step, we ran models stratified based on race. Unstandardized regression coefficient (b), standard error (SE), 95\% Confidence Intervals (CI), and $p$ values were reported.

\section{Results}

Table 1 presents the results of descriptive analysis in the pooled sample and also based on race. Compared to Whites, Blacks were younger, more frequently women, and had lower education and income. Blacks had worse CMC, SRH and BMI compared to Whites. Blacks also had higher positive affect at baseline compared to Whites. Blacks and Whites were similar in negative affect at baseline.

Table 2 presents the correlation matrix of the study variables in Whites and Blacks. While positive affect in wave 1 and 2 were correlated in both racial groups, the magnitude of the correlation between positive and negative affect were stronger for Whites than Blacks. Age was correlated with wave 1 positive and negative affect in Whites but not Blacks. Education and income also showed better correlations with wave 1 and wave 2 positive and negative affect in Whites than Blacks.

Table 3a summarizes the results of four linear regression models to test the effects of negative affect in wave 1 on positive affect in wave 1. Model 1-a only included demographic and socioeconomic factors without any interaction term. Model 2-a included demographic and socioeconomic factors, however also added the race by negative affect in wave 1 interaction term. Model 3-a included health factors as controls with no interaction term. Model 4-a included health factors as controls, with race by negative affect in wave 1 interaction term. According to our models, baseline negative affect showed strong inverse correlation with wave 1 positive affect, net of socioeconomic (Model 1-a) and health status (Model 3-a). Significant difference was found between Blacks and Whites for the association between positive and negative affect such that the inverse association was weaker for Blacks compared 
to Whites in a model with demographic and socioeconomic (Model 2-a) as well as health status (Model 4-a) (Table 3a).

Table 1. Descriptive statistics in the pooled sample and based on race.

\begin{tabular}{|c|c|c|c|c|c|c|c|}
\hline & & All & & Whites & & Blacks & $p$ \\
\hline & $\mathbf{N}$ & $\%$ & $\mathbf{N}$ & $\%$ & $\mathbf{N}$ & $\%$ & \\
\hline \multicolumn{8}{|l|}{ Demographics } \\
\hline \multicolumn{8}{|l|}{ Gender } \\
\hline Men & 3395 & 47.8 & 2683 & 47.9 & 121 & 37.7 & $<0.001$ \\
\hline \multirow[t]{2}{*}{ Women } & 3632 & 51.1 & 2917 & 52.1 & 200 & 62.3 & \\
\hline & Mean & SD & Mean & SD & Mean & SD & \\
\hline Age & 46.38 & 13.00 & 47.30 & 12.92 & 44.42 & 12.54 & $<0.001$ \\
\hline \multicolumn{8}{|l|}{ Socioeconomics } \\
\hline Education & 6.77 & 2.49 & 6.90 & 2.47 & 6.22 & 2.47 & $<0.001$ \\
\hline Income & $26,773.24$ & $26,891.19$ & $27,326.10$ & $27,509.71$ & $20,762.54$ & $19,730.37$ & $<0.001$ \\
\hline Self-Rated Health & 7.45 & 1.62 & 7.46 & 1.60 & 7.65 & 1.84 & 0.043 \\
\hline $\begin{array}{l}\text { Chronic Medical } \\
\text { Conditions }\end{array}$ & 2.41 & 2.51 & 2.39 & 2.46 & 2.53 & 2.96 & 0.422 \\
\hline Body Mass Index & 26.67 & 5.29 & 26.55 & 5.17 & 28.69 & 6.38 & $<0.001$ \\
\hline \multicolumn{8}{|l|}{ Outcomes } \\
\hline Negative Affect, Wave 1 & 1.53 & 0.62 & 1.53 & 0.61 & 1.53 & 0.65 & 0.879 \\
\hline Negative Affect, Wave 2 & 1.51 & 0.58 & 1.50 & 0.56 & 1.67 & 0.83 & 0.001 \\
\hline Positive Affect, Wave 1 & 3.39 & 0.73 & 3.38 & 0.72 & 3.52 & 0.77 & 0.002 \\
\hline Positive Affect, Wave 2 & 3.43 & 0.71 & 3.42 & 0.70 & 3.55 & 0.79 & 0.073 \\
\hline
\end{tabular}

Table $3 \mathrm{~b}$ provides a summary of four linear regression models with wave 1 positive affect as the independent variable and wave 1 negative affect as the dependent variable. Baseline positive affect showed strong inverse correlation with wave 1 negative affect, net of socioeconomic (Model 1-b) and health status (Model 3-b). Significant difference was found between Blacks and Whites for the association between positive and negative affect such that the inverse association was weaker for Blacks compared to Whites in the presence of demographic and socioeconomic (Model 2-b) as well as health status (Model 4-b) (Table 3b).

Table $4 \mathrm{a}$ presents the results of four linear regression models with wave 1 negative affect as the predictor and wave 2 positive affect as the outcome. According to our models, baseline negative affect showed strong inverse correlation with wave 1 positive affect, net of demographic and socioeconomic (Model 1-a) as well as health status (Model 3-a). Significant difference was found between Blacks and Whites for the association between positive and negative affect such that the inverse association was weaker for Blacks compared to Whites net of demographic and socioeconomic (Model 2-a) as well as health status (Model 4-a) (Table 4a).

Table $4 \mathrm{~b}$ shows the results of four linear regression models with wave 2 negative affect as the outcome. According to our models, baseline positive affect showed inverse correlation with wave 2 negative affect, net of demographic and socioeconomic (Model 1-b) and health status (Model 3-b). Significant difference was found between Blacks and Whites for the association between positive and negative affect such that the inverse association was weaker for Blacks compared to Whites net of demographic and socioeconomic (Model 2-b) as well as health status (Model 4-b) (Table 4b).

Table 5 summarizes predictors of wave 1 (Table 5a) positive and (Table 5a) negative affect in Whites and Blacks. Based on this table, net of demographic and socioeconomic as well as health status, positive affect showed inverse correlation with negative affect among Whites and Blacks. Regression coefficients were larger for Whites than Blacks.

Table 6 presents predictors of wave 2 positive (Table 6a) and negative (Table $6 \mathrm{~b}$ ) affect in Whites and Blacks. Based on this table, net of demographic and socioeconomic as well as health status, wave 1 positive affect showed inverse correlation with wave 2 negative affect among Whites but not Blacks. Wave 1 negative affect also showed inverse correlation with wave 2 positive affect among Whites but not Blacks. 
Table 2. Correlation matrix of the study variables in White and Black Americans.

\begin{tabular}{ccccccccccccc}
\hline & $\mathbf{1}$ & $\mathbf{2}$ & $\mathbf{3}$ & $\mathbf{4}$ & $\mathbf{5}$ & $\mathbf{6}$ & $\mathbf{7}$ & $\mathbf{8}$ & $\mathbf{9}$ & $\mathbf{1 0}$ & $\mathbf{1 1}$ \\
\hline 1 Gender (Women) & 1 & 0.01 & $-0.10^{* *}$ & $-0.36^{* *}$ & 0.00 & $0.12^{* *}$ & $-0.10^{* *}$ & $0.08^{* *}$ & $0.08^{* *}$ & $-0.03^{*}$ & -0.02 \\
\hline 2 Age & $-0.12^{*}$ & 1 & $-0.11^{* *}$ & $-0.15^{* *}$ & $-0.03^{*}$ & $0.18^{* *}$ & $0.11^{* *}$ & $-0.10^{* *}$ & $-0.10^{* *}$ & $0.10^{* *}$ & $0.14^{* *}$ \\
\hline 3 Education & -0.03 & -0.10 & 1 & $0.36^{* *}$ & $0.10^{* *}$ & $-0.13^{* *}$ & $-0.10^{* *}$ & $-0.09^{* *}$ & $-0.13^{* *}$ & 0.02 & $0.04^{*}$ \\
\hline 4 Income & $-0.26^{* *}$ & -0.04 & $0.36^{* *}$ & 1 & $0.10^{* *}$ & $-0.16^{* *}$ & 0.00 & $-0.10^{* *}$ & $-0.14^{* *}$ & $0.04^{* *}$ & $0.07^{* *}$ \\
\hline 5 Self-Rated Health & -0.07 & -0.02 & 0.02 & $0.17^{* *}$ & 1 & $-0.42^{* *}$ & $-0.24^{* *}$ & $-0.36^{* *}$ & $-0.28^{* *}$ & $0.39^{* *}$ & $0.31^{* *}$ \\
\hline 6 Chronic Medical Conditions & $0.13^{*}$ & $0.13^{*}$ & -0.10 & $-0.20^{* *}$ & $-0.33^{* *}$ & 1 & $0.17^{* *}$ & $0.41^{* *}$ & $0.30^{* *}$ & $-0.33^{* *}$ & $-0.24^{* *}$ \\
\hline 7 Body Mass Index & $0.14^{*}$ & 0.09 & -0.08 & -0.09 & $-0.25^{* *}$ & $0.15^{* *}$ & 1 & $0.06^{* *}$ & $0.06^{* *}$ & $-0.05^{* *}$ & $-0.06^{* *}$ \\
\hline 8 Negative Affect, Wave 1 & $0.15^{* *}$ & -0.05 & -0.05 & $-0.15^{* *}$ & $-0.21^{* *}$ & $0.27^{* *}$ & 0.10 & 1 & $0.51^{* *}$ & $-0.64^{* *}$ & $-0.39^{* *}$ \\
\hline 9 Negative Affect, Wave 2 & 0.12 & $-0.21^{*}$ & -0.15 & $-0.19^{*}$ & $-0.23^{* *}$ & 0.04 & $0.20^{*}$ & $0.40^{* *}$ & 1 & $-0.39^{* *}$ & $-0.62^{* *}$ \\
\hline 10 Positive Affect, Wave 1 & $-0.16^{* *}$ & 0.06 & -0.05 & 0.03 & $0.30^{* *}$ & $-0.18^{* *}$ & -0.05 & $-0.46^{* *}$ & $-0.21^{*}$ & 1 & $0.53^{* *}$ \\
\hline 11 Positive Affect, Wave 2 & -0.04 & $0.16^{*}$ & -0.05 & 0.03 & $0.44^{* *}$ & -0.04 & -0.13 & $-0.28^{* *}$ & $-0.44^{* *}$ & $0.51^{* *}$ & 1 \\
\hline
\end{tabular}

Whites, up diagonal; Blacks, low diagonal. ${ }^{*} p<0.05 ; * * p<0.01$. 
Table 3. Predictors of wave 1 positive and negative affect in the pooled sample.

\begin{tabular}{|c|c|c|c|c|c|c|c|c|c|c|c|c|}
\hline & B(SE) & $95 \%$ CI & $P$ & B(SE) & $95 \%$ CI & $P$ & B(SE) & $95 \%$ CI & $P$ & B(SE) & $95 \%$ CI & $P$ \\
\hline \multicolumn{13}{|l|}{ (a) } \\
\hline \multicolumn{13}{|l|}{ Outcome: Wave 1 Positive Affect } \\
\hline & & Model 1- $a$ & & & Model 2- $a$ & & & Model 3-a & & & Model 4- $a$ & \\
\hline Race (Blacks) & $0.14(0.03)$ & $0.07-0.20$ & $<0.001$ & $-2.34(0.15)$ & $-2.64--2.06$ & $<0.001$ & $0.12(0.03)$ & $0.06-0.19$ & $<0.001$ & $-2.23(0.07)$ & $-2.52--1.94$ & $<0.001$ \\
\hline Age & $0.00(0.00)$ & $0.00-0.00$ & 0.002 & $0.00(0.00)$ & $0.00-0.00$ & 0.001 & $0.00(0.00)$ & $0.00-0.00$ & $<0.001$ & $0.00(0.00)$ & $0.00-0.00$ & $<0.001$ \\
\hline Gender & $0.02(0.02)$ & $-0.01-0.05$ & 0.242 & $0.03(0.02)$ & $0.00-0.06$ & 0.070 & $0.01(0.02)$ & $-0.02-0.04$ & 0.489 & $0.02(0.01)$ & $-0.01-0.05$ & 0.198 \\
\hline Education & $-0.01(0.00)$ & $-0.01-0.00$ & 0.056 & $-0.00(0.00)$ & $-0.01-0.00$ & 0.096 & $-0.01(0.00)$ & $-0.01-0.00$ & 0.005 & $-0.01(0.00)$ & $-0.01-0.00$ & 0.011 \\
\hline Income & $-0.00(0.00)$ & $0.00-0.00$ & 0.321 & $-0.00(0.00)$ & $0.00-0.00$ & 0.435 & $-0.00(0.00)$ & $0.00-0.00$ & 0.080 & $-0.00(0.00)$ & $0.00-0.00$ & 0.125 \\
\hline Self-Rated Health & & & & & & & $0.08(0.01)$ & $0.07-0.09$ & $<0.001$ & $0.08(0.00)$ & $0.07-0.09$ & $<0.001$ \\
\hline Chronic Medical Conditions & & & & & & & $-0.01(0.00)$ & $-0.020--0.00$ & 0.002 & $-0.01(0.00)$ & $-0.02--0.00$ & 0.002 \\
\hline Body Mass Index & & & & & & & $-0.00(0.00)$ & $-0.00-0.01$ & 0.195 & $-0.00(0.00)$ & $-0.00-0.01$ & 0.147 \\
\hline Negative Affect, Wave 1 & $-0.76(0.01)$ & $-0.78--0.74$ & $<0.001$ & $-0.74(0.01)$ & $-0.76--0.71$ & $<0.001$ & $-0.67(0.01)$ & $-0.70--0.64$ & $<0.001$ & $-0.65(0.01)$ & $-0.68--0.62$ & $<0.001$ \\
\hline Negative Affect $\times$ Black & & & & $0.70(0.04)$ & $0.62-0.78$ & $<0.001$ & & & & $0.67(0.04)$ & $0.59-0.75$ & $<0.001$ \\
\hline \multicolumn{13}{|l|}{ (b) } \\
\hline \multicolumn{13}{|l|}{ Outcome: Wave 1 Negative Affect } \\
\hline & & Model 1-b & & & Model 2-b & & & Model 3-b & & & Model 4-b & \\
\hline Race (Blacks) & $0.04(0.03)$ & $-0.01-0.10$ & 0.136 & $-1.02(0.07)$ & $-1.15--0.89$ & $<0.001$ & $0.04(0.03)$ & $-0.01-0.10$ & 0.144 & $-0.95(0.07)$ & $-1.08--0.82$ & $<0.001$ \\
\hline Age & $0.00(0.00)$ & $0.00-0.00$ & $<0.001$ & $-0.00(0.00)$ & $0.00-0.00$ & $<0.001$ & $0.00(0.00)$ & $-0.01-0.00$ & $<0.001$ & $0.00(0.00)$ & $-0.01-0.00$ & $<0.001$ \\
\hline Gender & $0.04(0.01)$ & $0.01-0.07$ & 0.003 & $0.04(0.01)$ & $0.01-0.06$ & 0.006 & $0.02(0.01)$ & $-0.01-0.04$ & 0.156 & $0.01(0.01)$ & $-0.01-0.04$ & 0.243 \\
\hline Education & $-0.01(0.00)$ & $-0.02--0.01$ & $<0.001$ & $-0.01(0.00)$ & $-0.02--0.01$ & $<0.001$ & $-0.01(0.00)$ & $-0.01-0.00$ & $<0.001$ & $-0.01(0.00)$ & $-0.01-0.00$ & $<0.001$ \\
\hline Income & $0.00(0.00)$ & $0.00-0.00$ & $<0.001$ & $0.00(0.00)$ & $0.00-0.00$ & $<0.001$ & $0.00(0.00)$ & $0.00-0.00$ & 0.005 & $0.00(0.00)$ & $0.00-0.00$ & 0.006 \\
\hline Self-Rated Health & & & & & & & $-0.02(0.00)$ & $-0.03--0.01$ & $<0.001$ & $-0.02(0.00)$ & $-0.03--0.01$ & $<0.001$ \\
\hline Chronic Medical Conditions & & & & & & & $0.05(0.00)$ & $0.05-0.06$ & $<0.001$ & $0.05(0.00)$ & $0.05-0.06$ & $<0.001$ \\
\hline Body Mass Index & & & & & & & $0.00(0.00)$ & $0.00-0.00$ & 0.108 & $0.00(0.00)$ & $0.00-0.00$ & 0.057 \\
\hline Positive Affect, Wave 1 & $-0.54(0.01)$ & $-0.55--0.52$ & $<0.001$ & $-0.52(0.01)$ & $-0.54--0.50$ & $<0.001$ & $-0.45(0.01)$ & $-0.47--0.43$ & $<0.001$ & $-0.44(0.01)$ & $-0.46--0.42$ & $<0.001$ \\
\hline Positive Affect $\times$ Black & & & & $0.70(0.04)$ & $0.62-0.78$ & $<0.001$ & & & & $0.65(0.04)$ & $0.57-0.73$ & $<0.001$ \\
\hline
\end{tabular}


Table 4. Predictors of wave 2 positive and negative affect in the pooled sample.

\begin{tabular}{|c|c|c|c|c|c|c|c|c|c|c|c|c|}
\hline & $\mathrm{b}(\mathrm{SE})$ & $\begin{array}{c}95 \% \text { CI for } \\
\text { b }\end{array}$ & $P$ & $\mathrm{~b}(\mathrm{SE})$ & $\begin{array}{c}95 \% \text { CI for } \\
\text { b }\end{array}$ & $P$ & b(SE) & $95 \%$ CI for b & $P$ & $\mathrm{~b}(\mathrm{SE})$ & $95 \%$ CI for b & $P$ \\
\hline \multicolumn{13}{|l|}{ (a) } \\
\hline \multicolumn{13}{|l|}{ Outcome: Wave 2 Positive Affect } \\
\hline & & Model 1- $a$ & & & Model 2-a & & & Model 3-a & & & Model 4-a & \\
\hline Race (Blacks) & $0.16(0.06)$ & $0.05-0.27$ & 0.005 & $-0.96(0.27)$ & $-1.48--0.43$ & $<0.001$ & $0.10(0.06)$ & $-0.01-0.21$ & 0.072 & $-0.87(0.26)$ & $-10.39--0.35$ & 0.001 \\
\hline Age & $0.01(0.00)$ & $0.00-0.01$ & $<0.001$ & $0.01(0.00)$ & $0.00-0.01$ & $<0.001$ & $0.01(0.00)$ & $0.00-0.01$ & $<0.001$ & $-0.01(0.00)$ & $0.00-0.01$ & $<0.001$ \\
\hline Gender (Female) & $0.05(0.02)$ & $0.01-00.10$ & 0.027 & $0.05(0.02)$ & $0.01-0.10$ & 0.019 & $0.04(0.02)$ & $0.00-0.09$ & 0.054 & $0.05(0.02)$ & $0.00-0.09$ & 0.039 \\
\hline Education & $0.00(0.00)$ & $-0.01-0.01$ & 0.594 & $0.00(0.00)$ & $-0.01-0.01$ & 0.590 & $0.00(0.00)$ & $-0.01-0.01$ & 0.854 & $0.00(0.00)$ & $-00.01-00.01$ & 0.866 \\
\hline Income (\$) & $0.00(0.00)$ & $0.00-00.00$ & 0.003 & $0.00(0.00)$ & $0.00-0.00$ & 0.003 & $0.00(0.00)$ & $0.00-0.00$ & 0.018 & $0.00(0.00)$ & $0.00-0.00$ & 0.014 \\
\hline Self-Rated Health (1-10) & & & & & & & $0.09(0.01)$ & $0.08-0.11$ & $<0.001$ & $0.09(00.01)$ & $0.08-0.11$ & $<0.001$ \\
\hline Chronic Medical Conditions (n) & & & & & & & $0.02(0.01)$ & $-0.03--0.01$ & 0.002 & $-00.02(0.01)$ & $-0.03--0.01$ & 0.002 \\
\hline Body Mass Index (lb/in2) & & & & & & & $0.00(0.00)$ & $0.00-0.01$ & 0.679 & $0.00(0.00)$ & $0.00-0.01$ & 0.668 \\
\hline Negative Affect, Wave 1 & $-00.45(0.02)$ & $-0.49--0.42$ & $<0.001$ & $-00.45(0.02)$ & $-0.48--0.41$ & $<0.001$ & $-0.35(0.02)$ & $-0.39--0.31$ & $<0.001$ & $-00.35(0.02)$ & $-0.39--0.31$ & $<0.001$ \\
\hline Negative Affect $\times$ Black & & & & $0.31(0.07)$ & $0.17-0.46$ & $<0.001$ & & & & $0.27(0.07)$ & $0.13-0.42$ & $<0.001$ \\
\hline \multicolumn{13}{|l|}{ (b) } \\
\hline \multicolumn{13}{|l|}{ Outcome: Wave 2 Negative Affect } \\
\hline & & Model 1-b & & & Model 2-b & & & Model 3-b & & & Model 4-b & \\
\hline Race (Blacks) & $0.17(0.05)$ & $0.08-00.26$ & $<0.001$ & $-00.26(0.10)$ & $-0.46--0.05$ & 0.013 & $0.17(0.05)$ & $0.08-0.26$ & $<0.001$ & $-00.23(0.10)$ & $-0.43--0.03$ & 0.024 \\
\hline Age & $0.00(0.00)$ & $-0.01-0.00$ & $<0.001$ & $0.00(0.00)$ & $-0.01-0.00$ & $<0.001$ & $-0.01(0.00)$ & $-0.01-0.00$ & $<0.001$ & $-00.01(0.00)$ & $-0.01-0.00$ & $<0.001$ \\
\hline Gender (Female) & $0.02(0.02)$ & $-0.01-0.06$ & 0.205 & $0.02(0.02)$ & $-0.01-0.06$ & 0.242 & $0.01(0.02)$ & $-0.03-0.04$ & 0.758 & $0.00(0.02)$ & $-0.03-0.04$ & 0.824 \\
\hline Education & $-00.02(0.00)$ & $-0.03--0.01$ & $<0.001$ & $-00.02(0.00)$ & $-0.03--0.01$ & $<0.001$ & $-00.02(0.00)$ & $-0.03--0.01$ & $<0.001$ & $-00.02(0.00)$ & $-0.03--0.01$ & $<0.001$ \\
\hline Income (\$) & $0.00(0.00)$ & $0.00-00.00$ & $<0.001$ & $0.00(0.00)$ & $0.00-0.00$ & $<0.001$ & $0.00(0.00)$ & $0.00-0.00$ & $<0.001$ & $0.00(0.00)$ & $0.00-0.00$ & $<0.001$ \\
\hline Self-Rated Health (1-10) & & & & & & & $-0.03(0.01)$ & $-0.05--0.02$ & $<0.001$ & $-0.03(0.01)$ & $-0.04--0.02$ & $<0.001$ \\
\hline Chronic Medical Conditions (n) & & & & & & & $0.04(0.00)$ & $0.03-0.05$ & $<0.001$ & $0.04(0.00)$ & $0.03-0.05$ & $<0.001$ \\
\hline Body Mass Index (lb/in2) & & & & & & & $0.00(0.00)$ & $0.00-0.00$ & 0.665 & $0.01(0.00)$ & $0.00-0.00$ & 0.654 \\
\hline Positive Affect, Wave 1 & $-0.30(0.01)$ & $-0.32--0.27$ & $<0.001$ & $-0.29(0.01)$ & $-0.31--0.27$ & $<0.001$ & $-0.23(0.01)$ & $-0.26--0.20$ & $<0.001$ & $-0.23(0.01)$ & $-0.25--0.20$ & $<0.001$ \\
\hline Positive Affect $\times$ Black & & & & $0.28(0.06)$ & $0.16-0.40$ & $<0.001$ & & & & $0.26(0.06)$ & $0.15-0.38$ & $<0.001$ \\
\hline
\end{tabular}


Table 5. Predictors of wave 1 positive and negative affect in White and Black Americans.

\begin{tabular}{|c|c|c|c|c|c|c|c|c|c|c|c|c|}
\hline & B(SE) & $95 \%$ CI & $P$ & B(SE) & $95 \% \mathrm{CI}$ & $P$ & B(SE) & $95 \% \mathrm{CI}$ & $P$ & B(SE) & $95 \% \mathrm{CI}$ & $P$ \\
\hline & & & & Whites & & & & & & Blacks & & \\
\hline \multicolumn{13}{|l|}{ (a) } \\
\hline \multicolumn{13}{|l|}{ Outcome: Wave 1 Positive Affect } \\
\hline & & Model 1 & & & Model 2 & & & Model 3 & & & Model 4 & \\
\hline Age & $0.00(0.00)$ & $0.00-0.00$ & 0.002 & $0.00(0.00)$ & $0.00-0.00$ & $<0.001$ & $0.00(0.00)$ & $-0.01-0.01$ & 0.802 & $0.00(0.00)$ & $0.00-0.01$ & 0.447 \\
\hline Gender (Female) & $0.03(0.02)$ & $0.00-0.06$ & 0.058 & $0.02(0.02)$ & $-0.01-0.05$ & 0.198 & $-0.22(0.09)$ & $-0.38--0.05$ & 0.013 & $-0.18(0.09)$ & $-0.36--0.01$ & 0.041 \\
\hline Education & $-0.01(.00)$ & $0-0.01-0.00$ & 0.069 & $0-0.01(.00)$ & $-0.02-0.00$ & 0.006 & $-0.01(0.02)$ & $-0.05-0.02$ & 0.467 & $-0.01(0.02)$ & $-0.04-0.03$ & 0.642 \\
\hline Income (USD1000) & $0.00(0.00)$ & $0.00-0.00$ & 0.505 & $0.00(0.00)$ & $0.00-0.00$ & 0.178 & $0.00(0.00)$ & $0.00-0.00$ & 0.232 & $0.00(0.00)$ & $0.00-0.00$ & 0.086 \\
\hline Self-Rated Health (1-10) & & & & $0.08(0.01)$ & $0.07-0.09$ & $<0.001$ & & & & $0.08(0.02)$ & $0.04-0.13$ & 0.001 \\
\hline Chronic Medical Conditions ( $\mathrm{n}$ ) & & & & $-0.01(0.00)$ & $-0.02-0.00$ & 0.004 & & & & $-0.01(0.02)$ & $-0.04-0.02$ & 0.352 \\
\hline Body Mass Index (lb/in2) & & & & $0.00(0.00)$ & $0.00-0.00$ & 0.210 & & & & $0.00(0.01)$ & $-0.01-0.02$ & 0.666 \\
\hline Negative Affect, Wave 1 & $-0.77(0.01)$ & $-0.80--0.75$ & 0.000 & $-0.68(0.01)$ & $-0.71--0.65$ & $<0.001$ & $-0.55(0.06)$ & $-0.67--0.43$ & $<0.001$ & $-0.49(0.07)$ & $-0.62--0.36$ & $<0.001$ \\
\hline \multicolumn{13}{|l|}{ (b) } \\
\hline \multicolumn{13}{|l|}{ Outcome: Wave 1 Negative Affect } \\
\hline & & Model 1 & & & Model 2 & & & Model 3 & & & Model 4 & \\
\hline Age & $0.00(0.00)$ & $0.00-0.00$ & $<0.001$ & $0.00(0.00)$ & $-0.01-0.00$ & $<0.001$ & $0.00(0.00)$ & $-0.01-0.00$ & 0.467 & $0.00(0.00)$ & $-0.01-0.00$ & 0.219 \\
\hline Gender (Female) & $0.04(0.01)$ & $0.02-0.07$ & 0.001 & $0.02(0.01)$ & $0.00-0.05$ & 0.113 & $0.01(0.07)$ & $-0.13-0.16$ & 0.841 & $-0.01(0.08)$ & $-0.16-0.014$ & 0.923 \\
\hline Education & $-0.01(0.00)$ & $-0.02--0.01$ & $<0.001$ & $-0.01(0.00)$ & $-0.01-0.00$ & $<0.001$ & $0.00(0.01)$ & $-0.03-0.03$ & 0.831 & $-0.01(0.01)$ & $-0.03-0.02$ & 0.709 \\
\hline Income (\$) & $0.00(0.00)$ & $0.00-0.00$ & $<0.001$ & $0.00(0.00)$ & $0.00-0.00$ & 0.012 & $0.00(0.00)$ & $0.00-0.00$ & 0.018 & $0.00(0.00)$ & $0.00-0.00$ & 0.114 \\
\hline Self-Rated Health (1-10) & & & & $-0.02(0.00)$ & $-0.03-0.01$ & $<0.001$ & & & & $-0.02(0.02)$ & $-0.06-0.02$ & 0.378 \\
\hline Chronic Medical Conditions ( $\mathrm{n}$ ) & & & & $0.05(0.00)$ & $0.05-0.06$ & $<0.001$ & & & & $0.04(0.01)$ & $0.02-0.07$ & 0.001 \\
\hline Body Mass Index (lb/in2) & & & & $0.00(0.00)$ & $0.00-0.00$ & 0.059 & & & & $0.00(0.01)$ & $-0.01-0.01$ & 0.560 \\
\hline Positive Affect, Wave 1 & $-0.55(0.01)$ & $-0.56--0.53$ & $<0.001$ & $-0.46(0.01)$ & $-0.48--0.44$ & $<0.001$ & $-0.40(0.04)$ & $-0.48--0.31$ & $<0.001$ & $-0.35(0.05)$ & $-0.44--0.26$ & $<0.001$ \\
\hline
\end{tabular}


Table 6. Predictors of wave 1 positive and negative affect in White and Black Americans.

\begin{tabular}{|c|c|c|c|c|c|c|c|c|c|c|c|c|}
\hline & B(SE) & $95 \%$ CI & $P$ & B(SE) & $95 \%$ CI & $P$ & B(SE) & $95 \%$ CI & $P$ & B(SE) & $95 \%$ CI & $P$ \\
\hline (a) & & & & & & & & & & & & \\
\hline \multicolumn{13}{|l|}{ Outcome: Wave 2 Positive Affect } \\
\hline & & Model 1 & & & Model 2 & & & Model 3 & & & Model 4 & \\
\hline Age & $0.01(0.00)$ & $0.00-0.01$ & $<0.001$ & $0.01(0.00)$ & $0.00-0.01$ & $<0.001$ & $0.00(0.01)$ & $-0.01-0.01$ & 0.381 & $0.00(0.01)$ & $-0.01-0.01$ & 0.683 \\
\hline Gender (Female) & $0.04(0.02)$ & $0.00-0.08$ & 0.075 & $0.04(0.02)$ & $-0.01-0.08$ & 0.100 & $0.13(0.14)$ & $-0.14-0.41$ & 0.335 & $0.06(0.13)$ & $-0.20-0.33$ & 0.628 \\
\hline Education & $0.01(0.00)$ & $0.00-0.01$ & 0.224 & $0.00(.00)$ & $-0.01-0.01$ & 0.546 & $-0.02(0.02)$ & $-0.07-0.03$ & 0.351 & $-0.02(0.02)$ & $-0.06-0.03$ & 0.492 \\
\hline Income (USD1000) & $0.00(0.00)$ & $0.00-0.00$ & $<0.001$ & $0.00(.00)$ & $0.00-0.00$ & 0.001 & $0.00(0.00)$ & $0.00-0.00$ & 0.583 & $0.00(0.00)$ & $0.00-0.00$ & 0.655 \\
\hline Self-Rated Health (1-10) & & & & $0.05(0.01)$ & $0.03-0.06$ & $<0.001$ & & & & $0.15(0.04)$ & $0.08-0.23$ & 0.000 \\
\hline Chronic Medical Conditions (n) & & & & $-0.02(0.01)$ & $-0.03--0.01$ & 0.002 & & & & $0.06(0.02)$ & $0.01-0.11$ & 0.015 \\
\hline Body Mass Index (lb/in2) & & & & $0.00(0.00)$ & $0.00-0.00$ & 0.911 & & & & $-0.01(0.01)$ & $-0.03-0.01$ & 0.333 \\
\hline Positive Affect, Wave 1 & $0.46(0.02)$ & $0.43-0.50$ & $<0.001$ & $0.43(0.02)$ & $0.39-0.47$ & $<0.001$ & $0.51(0.09)$ & $0.33-0.69$ & $<0.001$ & $0.43(0.09)$ & $0.26-0.61$ & $<0.00$ \\
\hline Negative Affect, Wave 1 & $-0.09(0.02)$ & $-0.14--0.05$ & $<0.001$ & $-0.06(0.02)$ & $-0.10--0.01$ & 0.020 & $-0.05(0.09)$ & $-0.23-0.13$ & 0.578 & $-0.06(0.09)$ & $-0.23-0.12$ & 0.504 \\
\hline \multicolumn{13}{|l|}{ (b) } \\
\hline \multicolumn{13}{|l|}{ Outcome: Wave 2 Negative Affect } \\
\hline & & Model 1 & & & Model 2 & & & Model 3 & & & Model 4 & \\
\hline Age & $0.00(0.00)$ & $0.00-0.00$ & $<0.001$ & $0.00(0.00)$ & $0.00-0.00$ & $<0.001$ & $-0.01(0.01)$ & $-0.02-0.00$ & 0.044 & $-0.01(0.01)$ & $-0.02-0.00$ & 0.049 \\
\hline Gender (Female) & $0.01(0.02)$ & $-0.03-0.04$ & 0.639 & $0.00(0.02)$ & $-0.04-0.03$ & 0.900 & $0.05(0.15)$ & $-0.26-0.35$ & 0.764 & $0.02(0.16)$ & $-0.29-0.34$ & 0.876 \\
\hline Education & $-0.02(0.00)$ & $-0.03--0.01$ & $<0.001$ & $-0.02(0.00)$ & $-0.02--0.01$ & $<0.001$ & $-0.03(0.03)$ & $-0.08-0.02$ & 0.266 & $-0.03(0.03)$ & $-0.08-0.03$ & 0.310 \\
\hline Income (\$) & $0.00(0.00)$ & $0.00-0.00$ & $<0.001$ & $0.00(0.00)$ & $0.00-0.00$ & $<0.001$ & $0.00(0.00)$ & $0.00-0.00$ & 0.395 & $0.00(0.00)$ & $0.00-0.00$ & 0.368 \\
\hline Self-Rated Health (1-10) & & & & $-0.02(0.01)$ & $-0.04--0.01$ & $<0.001$ & & & & $-0.04(0.05)$ & $-0.13-0.06$ & 0.433 \\
\hline Chronic Medical Conditions (n) & & & & $0.02(0.00)$ & $0.01-0.03$ & $<0.001$ & & & & $-0.03(0.03)$ & $-0.08-0.03$ & 0.348 \\
\hline Body Mass Index (lb/in2) & & & & $0.00(0.00)$ & $0.00-0.00$ & 0.915 & & & & $0.02(0.01)$ & $0.00-0.04$ & 0.057 \\
\hline Negative Affect, Wave 1 & $0.41(0.02)$ & $0.37-0.45$ & $<0.001$ & $0.36(0.02)$ & $0.32-0.40$ & $<0.001$ & $0.36(0.10)$ & $0.16-0.56$ & 0.001 & $0.37(0.11)$ & $0.17-0.58$ & 0.001 \\
\hline Positive Affect, Wave 1 & $-0.08(0.02)$ & $-0.11--0.05$ & $<0.001$ & $-0.06(0.02)$ & $-0.10--0.03$ & $<0.001$ & $-0.03(0.10)$ & $-0.23-0.17$ & 0.775 & $-0.02(0.11)$ & $-0.24-0.20$ & 0.845 \\
\hline
\end{tabular}




\section{Discussion}

In our study, Black and White Americans differed in the magnitude of the inverse correlation between positive and negative affect at baseline and over time. According to our study, the negative association between positive and negative affect was weaker for Black compared to White Americans. These differential correlations remained significant at baseline and over time, net of demographic, socioeconomic, and health status.

Our finding of a stronger link between positive and negative affect among White compared to Black Americans is consistent with previous findings showing weaker negative association between depressive symptoms and hopefulness among Whites than Blacks [67]. In the presence of similar depressive symptoms, Blacks maintain higher levels of hope than Whites, [67] and Blacks who endorse high negative affect maintain high positive affect as well. Black-White differences also exist in the magnitude of correlation between depression and evaluation of self [68]. Negative affect and depressive symptoms differently negative feelings and cognitions about self in Whites and Blacks [68]. The weaker effects of depression and depressive symptoms on physical health outcomes, such as incident chronic disease [69] and all-cause [70] and disease specific chronic disease [71], in Whites than Blacks are also known. In one study, depressive symptoms predicted future clinical depression in Whites but not Blacks [30], and in another study, race altered how depressive symptoms map on clinical depression [46].

Canady et al. found the item "I was happy" as the only item with different loadings between Blacks and Whites after applying the cross-group constraints [43]. In a recent study, Assari and Moazen-Zadeh found differences for several positive affect items, including the item "I was happy," on a depression scale for Blacks and Whites. The study showed worse item loadings for positive items, namely "as good" and "hopeful," in Blacks than Whites. In the final model with a very good fit to the data, the item "as good" showed poor loading for Whites and Blacks, however, the item "hopeful" showed good loading for Whites but poor loading for Blacks [42].

A recent body of evidence has shown major racial differences in socioeconomic and health correlates of negative emotions [51-53]. Negative emotions better predict medical conditions, obesity, and mortality in Whites than Blacks [52,53]. Based on Black-White health paradox, defined as less frequent depression despite a higher prevalence of chronic medical conditions among Blacks compared to Whites [54,72-82], adversities and negative emotions are more common among Blacks [69]. Our findings may explain why emotional disorders are not as common as expected in Blacks.

According to the "undoing hypothesis," positive emotions are able to undo the harmful effects of negative emotions [5,7]. Maintaining higher levels of positive affect in the presence of negative affect in Blacks compared to Whites may explain the weaker effects of negative emotions on physical health of Blacks. In this view, positive affect operates as a buffer against harms associated with negative affect $[7,20]$. This phenomenon can explain why depressive symptoms predict incident chronic disease [69] and mortality [52,53] for Whites but not Blacks. Inflammation also better correlates with depression for Blacks than Whites [81,82]. This finding can also explain why depressive symptoms predict subsequent MDD among Whites but not Blacks [29]. This phenomenon may also explain why Blacks report better well-being (positive affect) than Whites, despite higher levels of stress, psychological distress, and depressive symptoms [83].

Our findings are in support of the literature that suggests positive and negative affect are separate but interconnected components of depression measurement, among both Blacks and Whites [25-27,44,84]. While this study investigated positive and negative emotions, there is a need to explore racial differences in correlations between emotions and other domains of depression such as somatic complaints and interpersonal problems [85-88]. Future research may also test if resources and assets such as self-esteem, social support, religion, or culture explain Black-White variation in the link between positive and negative affect.

Our findings on Black-White differences in the link between positive and negative affect have major implications for measurement of depression and depressive symptoms in ethnically diverse 
populations. Clinicians and researchers who work with racially diverse populations should be aware of race-specific links between positive and negative emotions. Compared to their White counterparts, Blacks with depression may endorse higher levels of positive affect, which may reduce chance of diagnosis of depression. We argue that positive and negative items of depression should not be simply summed among diverse populations. Instead, positive and negative domains should be considered as inter-connected but separate domains across racial groups. Domain-specific evaluation of mood may be a better option than calculating a sum score of positive and negative items. Considering such cross-ethnic variations may help with more accurate diagnosis and treatment of depression among ethnically diverse populations [85,89].

Our findings also have implications for epidemiological studies of depression across various racial groups. The differential link between positive and negative affect based on race causes measurement bias for cross-racial measurement of mood outcomes, including but not limited to depression. Programs that screen ethnically diverse samples for depression or depressive symptomatology should be aware of how race alters the concordance of positive and negative affect. There is still a need for further research on equivalence of depression, affect, and mood measurement among diverse racial and ethnic groups [51,90,91]. Future research should focus on cross-racial validation of measures of depression.

Our study is subject to at least three limitations. The major limitation of this study was not measuring culture [92,93]. Second, we did not include clinical diagnosis of MDD according to the DSM criteria (composite international diagnostic interview criteria were used). Third, we did not consider race by gender differences in this study. Despite how the race by gender intersection may shape experience and expression of positive and negative emotions [94], limited sample size of Blacks did not allow us to break our sample of Blacks to additional sub-groups. Despite this limitation, a unique strength of our study was using nationally representative data of American adults that resulted in nationally generalizable findings to the U.S. population.

In conclusion, our findings suggest that positive and negative affect have a weaker negative association in Blacks than Whites, suggesting that in the presence of similar negative affect, Blacks maintain higher levels of positive affect than Whites. These findings have health implications given the protective effect of positive affect (the "undoing hypothesis"). It is unknown whether racial differences in the magnitude of the correlation between positive and negative affect explains previously observed Black-White differences in psychosocial and medical correlates of depression. In addition, these findings are also important for measurement of depression. This finding calls into question the measurement equivalence of affect, mood, and depression outcomes among Blacks and Whites.

\section{Conclusions}

In summary, positive and negative affect are more concurrent in Blacks compared to Whites. This finding has implications for cross-racial measurement of affect and mood, including depression. This information may have implications for diagnosis and screening of depression in racially diverse populations.

Acknowledgments: Shervin Assari is supported by the Heinz C. Prechter Bipolar Research Fund and the Richard Tam Foundation at the University of Michigan Depression Center. Ethics: The study protocol was approved by the University of Michigan Institutional Review Board, and written informed consent was obtained for all participants. The study was funded by the National Institute on Aging (NIA). Monetary incentives were given at both Wave 1 and Wave 2 for compensation (US \$20 for completion of MIDUS 1 surveys and up to US \$60 for completion of MIDUS 2 surveys). Funding: This research was supported by a grant from the National Institute on Aging (P01-AG020166) to conduct a longitudinal follow-up of the MIDUS investigation. The original study was supported by the John D. and Catherine T. MacArthur Foundation Research Network on Successful Midlife Development.

Author Contributions: Shervin Assari was responsible for the design and analysis of the data. Maryam Moghani Lankarani drafted the manuscript.

Conflicts of Interest: Maryam Moghani Lankarani and Shervin Assari declare that they have no competing interests. 


\section{References}

1. Bradburn, N.M. The Structure of Psychological Well-Being; Aldine: Chicago, IL, USA, 1969.

2. Ryff, C.D.; Keyes, C.L. The structure of psychological well-being revisited. J. Personal. Soc. Psychol. 1995, 69, 719. [CrossRef]

3. Frijda, N.H. The Emotions; Cambridge University Press: Cambridge, UK, 1986.

4. Lazarus, R.S. Emotion and Adaptation; Oxford University Press: New York, NY, USA, 1991.

5. Fredrickson, B.L.; Levenson, R.W. Positive emotions speed recovery from the cardiovascular sequelae of negative emotions. Cogn. Emot. 1998, 12, 191-220. [CrossRef] [PubMed]

6. Fredrickson, B.L.; Maynard, K.E.; Helms, M.J.; Haney, T.L.; Siegler, I.C.; Barefoot, J.C. Hostility predicts magnitude and duration of blood pressure response to anger. J. Behav. Med. 2000, 23, 229-243. [CrossRef] [PubMed]

7. Fredrickson, B.L.; Mancuso, R.A.; Branigan, C.; Tugade, M.M. The Undoing Effect of Positive Emotions. Motiv. Emot. 2000, 24, 237-258. [CrossRef] [PubMed]

8. Watson, D.; Clark, L.A. Negative affectivity: The disposition to experience aversive emotional states. Psychol. Bull. 1984, 96, 465-490. [CrossRef] [PubMed]

9. Miller, D.J.; Vachon, D.D.; Lynam, D.R. Neuroticism, negative affect, and negative affect instability: Establishing convergent and discriminant validity using ecological momentary assessment. Personal. Individ. Differ. 2009, 47, 873-877. [CrossRef] [PubMed]

10. Clark, L.A.; Watson, D.; Mineka, S. Temperament, personality, and the mood and anxiety disorders. J. Abnorm. Psychol. 1994, 103, 103-116. [CrossRef] [PubMed]

11. Pressman, S.D.; Cohen, S. Does positive affect influence health? Psychol. Bull. 2005, 131, 925. [CrossRef] [PubMed]

12. Danner, D.D.; Snowdon, D.A.; Friesen, W.V. Positive emotions in early life and longevity: Findings from the nun study. J. Personal. Soc. Psychol. 2001, 80, 804. [CrossRef]

13. Hou, W.K.; Law, C.C.; Fu, Y.T. Does change in positive affect mediate and/or moderate the impact of symptom distress on psychological adjustment after cancer diagnosis? A prospective analysis. Psychol. Health 2010, 25, 417-431. [CrossRef] [PubMed]

14. Levenson, R.W. Emotion and the autonomic nervous system: A prospectus for research on autonomic specificity. In Social Psychophysiology and Emotion: Theory and Clinical Applications; Wagner, H.L., Ed.; Wiley: London, UK, 1988; pp. 17-42.

15. Kiecolt-Glaser, J.K.; McGuire, L.; Robles, T.F.; Glaser, R. Emotions, morbidity, and mortality: New perspectives from psychoneuroimmunology. Annu. Rev. Psychol. 2002, 53, 83-107. [CrossRef] [PubMed]

16. Levenson, R.W. Human emotion: A functional view. In The Nature of Emotion: Fundamental Questions; Ekman, P., Davidson, R., Eds.; Oxford University Press: New York, NY, USA, 1994; pp. 123-126.

17. Bares, C.B.; Andrade, F.H. Racial/ethnic differences in the longitudinal progression of co-occurring negative affect and cigarette use: From adolescence to young adulthood. Addict. Behav. 2012, 37, 632-640. [CrossRef] [PubMed]

18. Isen, A.M.; Daubman, K.A.; Nowicki, G.P. Positive affect facilitates creative problem solving. J. Personal. Soc. Psychol. 1987, 52, 1122-1131. [CrossRef]

19. Estrada, C.A.; Isen, A.M.; Young, M.J. Positive affect facilitates integration of information and decreases anchoring in reasoning among physicians. Organ. Behav. Hum. Decis. Process. 1997, 72, 117-135. [CrossRef]

20. Levenson, R.W.; Ekman, P.; Friesen, W.V. Voluntary facial action generates emotion-specific autonomic nervous system activity. Psychophysiology 1990, 27, 363-384. [CrossRef] [PubMed]

21. Fredrickson, B.L.; Joiner, T. Positive emotions trigger upward spirals toward emotional well-being. Psychol. Sci. 2002, 13, 172-175. [CrossRef] [PubMed]

22. Watson, D. Rethinking the mood and anxiety disorders: A quantitative hierarchical model for DSM-V. J. Abnorm. Psychol. 2005, 114, 522-536. [CrossRef] [PubMed]

23. Dua, J.K. The role of negative affect and positive affect in stress, depression, self-esteem, assertiveness, Type A behaviors, psychological health, and physical health. Genet. Soc. Gen. Psychol. Monogr. 1993, 119, 515-552. [PubMed] 
24. Garamoni, G.L.; Reynolds, C.F.; Thase, M.E.; Frank, E.; Berman, S.R.; Fasiczka, A.L. The balance of positive and negative affects in major depression: A further test of the states of mind model. Psychiatry Res. 1991, 39, 99-108. [CrossRef]

25. Nguyen, H.T.; Kitner-Triolo, M.; Evans, M.K.; Zonderman, A.B. Factorial invariance of the CES-D in low socioeconomic status African Americans compared with a nationally representative sample. Psychiatry Res. 2004, 126, 177-187. [CrossRef] [PubMed]

26. Hales, D.P.; Dishman, R.K.; Motl, R.W.; Addy, C.L.; Pfeiffer, K.A.; Pate, R.R. Factorial validity and invariance of the center for epidemiologic studies depression (CES-D) scale in a sample of black and white adolescent girls. Ethn. Dis. 2005, 16, 1-8.

27. Kim, G.; DeCoster, J.; Huang, C.H.; Chiriboga, D.A. Race/ethnicity and the factor structure of the Center for Epidemiologic Studies Depression Scale: A meta-analysis. Cult. Divers. Ethn. Minor. Psychol. 2011, 17, 381. [CrossRef] [PubMed]

28. Becker, A.E.; Kleinman, A. Mental health and the global agenda. N. Engl. J. Med. 2013, 369, 66-73. [CrossRef] [PubMed]

29. Moazen-Zadeh, E.; Assari, S. Depressive symptoms predict major depressive disorder after 15 years among Whites but not Blacks. Front. Public Health 2016, 4, 13. [CrossRef] [PubMed]

30. Jang, Y.; Kwag, K.H.; Chiriboga, D.A. Not saying I am happy does not mean I am not: Cultural influences on responses to positive affect items in the CES-D. J. Gerontol. B Psychol. Sci. Soc. Sci. 2010, 65, 684-690. [CrossRef] [PubMed]

31. Lovejoy, M.C.; Steuerwald, B.L. Subsyndromal unipolar and bipolar disorders: Comparisons on positive and negative affect. J. Abnorm. Psychol. 1995, 104, 381-384. [CrossRef] [PubMed]

32. Robins, L.N.; Wing, J.; Wittchen, H.U.; Helzer, J.E.; Babor, T.F.; Burke, J.; Farmer, A.; Jablenski, A.; Pickens, R.; Regier, D.A.; et al. The Composite International Diagnostic Interview: An epidemiologic instrument suitable for use in conjunction with different diagnostic systems and in different cultures. Arch. Gen. Psychiatry 1988, 45, 1069-1077. [CrossRef] [PubMed]

33. Wittchen, H.U. Reliability and validity studies of the WHO-Composite International Diagnostic Interview (CIDI): A critical review. J. Psychiatr. Res. 1994, 28, 57-84. [CrossRef]

34. Kessler, R.C.; Wittchen, H.U.; Abelson, J.M.; Mcgonagle, K.; Schwarz, N.; Kendler, K.S.; Knäuper, B.; Zhao, S. Methodological studies of the Composite International Diagnostic Interview (CIDI) in the US national comorbidity survey (NCS). Int. J. Methods Psychiatr. Res. 1998, 7, 33-55. [CrossRef]

35. Kessler, R.C.; Calabrese, J.R.; Farley, P.A.; Gruber, M.J.; Jewell, M.A.; Katon, W.; Keck, P.E.; Nierenberg, A.A.; Sampson, N.A.; Shear, M.K.; et al. Composite International Diagnostic Interview screening scales for DSM-IV anxiety and mood disorders. Psychol. Med. 2013, 43, 1625-1637. [CrossRef] [PubMed]

36. Assari, S.; Lankarani, M.M.; Moazen, B. Religious beliefs may reduce the negative effect of psychiatric disorders on age of onset of suicidal ideation among blacks in the United States. Int. J. Prev. Med. 2012, 3, 358-364. [PubMed]

37. Assari, S.; Lankarani, M.M.; Lankarani, R.M. Ethnicity modifies the effects of anxiety and drug use on suicidal ideation among Black adults in the United States. Int. J. Prev. Med. 2013, 4, 1251-1257. [PubMed]

38. Radloff, L.S. The CES-D scale a self-report depression scale for research in the general population. Appl. Psychol. Meas. 1977, 1, 385-401. [CrossRef]

39. Coman, E.N.; Iordache, E.; Schensul, J.J.; Coiculescu, I. Comparisons of CES-D depression scoring methods in two older adults ethnic groups. The emergence of an ethnic-specific brief three-item CES-D scale. Int. J. Geriatr. Psychiatry 2013, 28, 424-432. [CrossRef] [PubMed]

40. Skriner, L.C.; Chu, B.C. Cross-ethnic measurement invariance of the SCARED and CES-D in a youth sample. Psychol. Assess. 2014, 26, 332. [CrossRef] [PubMed]

41. Tran, T.V. Exploring the equivalence of factor structure in a measure of depression between Black and White women: Measurement issues in comparative research. Res. Soc. Work Pract. 1997, 7, 500-517. [CrossRef]

42. Assari, S.; Moazen-Zadeh, E. Confirmatory Factor Analysis of the 12-Item Center for Epidemiologic Studies Depression Scale among Blacks and Whites. Front. Psychiatry 2016, 7, 178. [CrossRef] [PubMed]

43. Canady, R.B.; Stommel, M.; Holzman, C. Measurement properties of the Centers for Epidemiological Studies Depression Scale (CES-D) in a sample of African-American and non-Hispanic White pregnant women. J. Nurs. Meas. 2009, 17, 91. [CrossRef] [PubMed] 
44. Boutin-Foster, C. An item-level analysis of the Center for Epidemiologic Studies Depression Scale (CES-D) by race and ethnicity in patients with coronary artery disease. Int. J. Geriatr. Psychiatry 2008, 23, 1034-1039. [CrossRef] [PubMed]

45. Assari, S.; Moazen-Zadeh, E. Ethnic Variation in the cross-sectional association between domains of depressive symptoms and clinical depression. Front. Psychiatry 2016, 7, 53. [CrossRef] [PubMed]

46. Watson, D.; Clark, L.A. Measurement and mismeasurement of mood: Recurrent and emergent issues. J. Personal. Assess. 1997, 68, 267-296. [CrossRef] [PubMed]

47. Roberts, R.E. Reliability of the CES-D scale in different ethnic contexts. Psychiatry Res. 1980, 2, 125-134. [CrossRef]

48. Aneshensel, C.S.; Clark, V.A.; Frerichs, R.R. Race, ethnicity, and depression: A confirmatory analysis. J. Personal. Soc. Psychol. 1983, 44, 385. [CrossRef]

49. Vera, M.; Alegría, M.; Freeman, D.; Robles, R.R.; Ríos, R.; Ríos, C.F. Depressive symptoms among Puerto Ricans: Island poor compared with residents of the New York City area. Am. J. Epidemiol. 1991, 134, 502-510. [CrossRef] [PubMed]

50. Cole, S.R.; Kawachi, I.; Maller, S.J.; Berkman, L.F. Test of item-response bias in the CES-D scale: Experience from the New Haven EPESE study. J. Clin. Epidemiol. 2000, 53, 285-289. [CrossRef]

51. Assari, S.; Sonnega, A.; Pepin, R.; Leggett, A. Residual effects of restless sleep over depressive symptoms on chronic medical conditions: Race by gender differences. J. Racial Ethn. Health Disparities 2017, 4, 59-69. [CrossRef] [PubMed]

52. Assari, S.; Moazen-Zadeh, E.; Lankarani, M.M.; Foster, V.M. Black White differences in residual effects of depressive symptoms over socioeconomics and physical health on all-cause mortality in the United States. Front. Public Health 2016, 4, 40. [CrossRef] [PubMed]

53. Assari, S.; Burgard, S. Black-White differences in the effect of baseline depressive symptoms on deaths due to renal diseases: 25 year follow up of a nationally representative community sample. J. Ren. Inj. Prev. 2015, 4, 127. [PubMed]

54. Williams, D.R.; Gonzalez, H.M.; Neighbors, H.; Nesse, R.; Abelson, J.M.; Sweetman, J.; Jackson, J.S. Prevalence and distribution of major depressive disorder in African Americans, Caribbean Blacks, and non-Hispanic Whites: Results from the National Survey of American Life. Arch. Gen. Psychiatry 2007, 64, 305-315. [CrossRef] [PubMed]

55. Merz, E.L.; Malcarne, V.L.; Roesch, S.C.; Ko, C.M.; Emerson, M.; Roma, V.G.; Sadler, G.R. Psychometric properties of Positive and Negative Affect Schedule (PANAS) original and short forms in an African American community sample. J. Affect. Disord. 2013, 151, 942-949. [CrossRef] [PubMed]

56. Crockett, L.J.; Randall, B.A.; Shen, Y.L.; Russell, S.T.; Driscoll, A.K. Measurement equivalence of the center for epidemiological studies depression scale for Latino and Anglo adolescents: A national study. J. Consult. Clin. Psychol. 2005, 73, 47-58. [CrossRef] [PubMed]

57. Black, H.K.; White, T.; Hannum, S.M. The lived experience of depression in elderly African American women. J. Gerontol. B Psychol. Sci. Soc. Sci. 2007, 62, S392-S398. [CrossRef] [PubMed]

58. Radler, B.T.; Ryff, C.D. Who participates? Accounting for longitudinal retention in the MIDUS national study of health and well-being. J. Aging Health 2010, 22, 307-331. [CrossRef] [PubMed]

59. Dienberg Love, G.; Seeman, T.E.; Weinstein, M.; Ryff, C.D. Bioindicators in the MIDUS national study: Protocol, measures, sample, and comparative context. J. Aging Health 2010, 22, 1059-1080. [CrossRef] [PubMed]

60. Midlife in the United States (MIDUS). Available online: http:/ / www.midus.wisc.edu (accessed on 7 July 2017).

61. National Institute on Aging Database of Longitudinal Studies. Available online: http:/ /www.nia.nih.gov/ ResearchInformation/ScientificResources/LongitudinalStudies.htm (accessed on 7 July 2017).

62. Snowden, M.; Dhingra, S.S.; Keyes, C.L.; Anderson, L.A. Changes in mental well-being in the transition to late life: Findings from MIDUS I and II. Am. J. Public Health 2010, 100, 2385-2388. [CrossRef] [PubMed]

63. Mroczek, D.K.; Kolarz, C.M. The effect of age on positive and negative affect: A developmental perspective on happiness. J. Pers. Soc. Psychol. 1998, 75, 1333-1349. [CrossRef] [PubMed]

64. Grzywacz, J.G. Work-family spillover and health during midlife: Is managing conflict everything? Am. J. Health Promot. 2000, 14, 236-243. [CrossRef] [PubMed] 
65. Keyes, C.L.M. Subjective change and its consequences for emotional well-being. Motiv. Emot. 2000, 24, 67-83. [CrossRef]

66. Walen, H.R.; Lachman, M.E. Social support and strain from partner, family, and friends: Costs and benefits for men and women in adulthood. J. Soc. Personal Relatsh. 2000, 17, 5-30. [CrossRef]

67. Assari, S.; Lankarani, M.M. Depressive symptoms are associated with more hopelessness among White than Black older adults. Front. Public Health 2016, 4, 82. [CrossRef] [PubMed]

68. Assari, S.; Dejman, M. Heterogeneity of the link between depression and self-esteem based on race. Casp. J. Neurol. Sci. 2016, 2, 7-21. [CrossRef]

69. Assari, S.; Burgard, S.; Zivin, K. Long-term reciprocal associations between depressive symptoms and number of chronic medical conditions: Longitudinal support for Black-White health paradox. J. Racial Ethn. Health Disparities 2015, 2, 589-597. [CrossRef] [PubMed]

70. Assari, S. Race and Ethnic Differences in Additive and Multiplicative Effects of Depression and Anxiety on Cardiovascular Risk. Int. J. Prev Med. 2016, 7, 22. [CrossRef] [PubMed]

71. Assari, S.; Lankarani, M.M. Chronic Medical Conditions and Negative Affect; Racial Variation in Reciprocal Associations Over Time. Front. Psychiatry 2016, 7, 140. [CrossRef]

72. Mouzon, D.M. Can family relationships explain the race paradox in mental health? J. Marriage Fam. 2013, 75, 470-485. [CrossRef]

73. Mouzon, D.M. Relationships of choice: Can friendships or fictive kinships explain the race paradox in mental health? Soc. Sci. Res. 2014, 44, 32-43. [CrossRef] [PubMed]

74. Breslau, J.; Aguilar-Gaxiola, S.; Kendler, K.S.; Su, M.; Williams, D.; Kessler, R.C. Specifying race-ethnic differences in risk for psychiatric disorder in a USA national sample. Psychol. Med. 2006, 36, 57-68. [CrossRef] [PubMed]

75. Breslau, J.; Kendler, K.S.; Su, M.; Gaxiola-Aguilar, S.; Kessler, R.C. Lifetime risk and persistence of psychiatric disorders across ethnic groups in the United States. Psychol. Med. 2005, 35, 317-327. [CrossRef]

76. Williams, D.R.; Yu, R.; Jackson, J.S.; Anderson, N.B. Racial differences in physical and mental health: Socioeconomic status, stress, and discrimination. J. Health Psychol. 1997, 2, 335-351. [CrossRef] [PubMed]

77. Zhang, A.Y.; Snowden, L.R. Ethnic characteristics of mental disorders in five U.S. communities. Cult. Divers. Ethn. Minor. Psychol. 1999, 5, 134-146. [CrossRef]

78. Kessler, R.C.; McGonagle, K.A.; Zhao, S.; Nelson, C.B.; Hughes, M.; Eshleman, S.; Kendler, S. Lifetime and 12-month prevalence of DSM-III-R psychiatric disorders in the United States: Results from the National Comorbidity Survey. Arch. Gen. Psychiatry 1994, 51, 8-19. [CrossRef] [PubMed]

79. Mouzon, D.M. Religious involvement and the Black-White paradox in mental health. Race Soc. Probl. 2017, 9, 63-78. [CrossRef]

80. Barnes, D.M.; Keyes, K.M.; Bates, L.M. Racial differences in depression in the United States: How do subgroup analyses inform a paradox? Soc. Psychiatry Psychiatr. Epidemiol. 2013, 48, 1941-1949. [CrossRef] [PubMed]

81. Stewart, J.C. One effect size does not fit all-Is the depression-inflammation link missing in racial/ethnic minority individuals? JAMA Psychiatry 2016, 73, 301-302. [CrossRef] [PubMed]

82. Case, S.M.; Stewart, J.C. Race/ethnicity moderates the relationship between depressive symptom severity and C-reactive protein: 2005-2010 NHANES data. Brain Behav. Immun. 2014, 41, 101-108. [CrossRef] [PubMed]

83. Ryff, C.D.; Keyes, C.L.; Hughes, D.L. Status inequalities, perceived discrimination, and eudaimonic well-being: Do the challenges of minority life hone purpose and growth? J. Health Soc. Behav. 2003, 44, 275-291. [CrossRef] [PubMed]

84. Callahan, C.M.; Wolinsky, F.D. The effect of gender and race on the measurement properties of the CES-D in older adults. Med. Care 1994, 32, 341-356. [CrossRef] [PubMed]

85. Angel, R.; Thoits, P. The impact of culture on the cognitive structure of illness. Cult. Med. Psychiatry 1987, 11, 465-494. [CrossRef] [PubMed]

86. Baker, F.M.; Okwumabua, J.; Philipose, V.; Wong, S. Screening African-American elderly for the presence of depressive symptoms: A preliminary investigation. J. Geriatr. Psychiatry Neurol. 1996, 9, 127-132. [CrossRef] [PubMed]

87. Dick, R.W.; Beals, J.; Keane, E.M.; Manson, S.M. Factorial structure of the CES-D among American Indian adolescents. J. Adolesc. 1994, 17, 73-79. [CrossRef] 
88. Miller, T.Q.; Markides, K.S.; Black, S.A. The factor structure of the CES-D in two surveys of elderly Mexican Americans. J. Gerontol. B Psychol. Sci. Soc. Sci. 1997, 52, S259-S269. [CrossRef] [PubMed]

89. McBarnette, L.S. African American women. SAGE Race Ethn. Relat. 1996, 15, $43-67$.

90. Yang, F.M.; Jones, R.N. Center for Epidemiologic Studies-Depression scale (CES-D) item response bias found with Mantel-Haenszel method was successfully replicated using latent variable modeling. J. Clin. Epidemiol. 2007, 60, 1195-1200. [CrossRef] [PubMed]

91. Kim, G.; Chiriboga, D.A.; Jang, Y. Cultural equivalence in depressive symptoms in older White, Black, and Mexican-American adults. J. Am. Geriatr. Soc. 2009, 57, 790-796. [CrossRef] [PubMed]

92. Mesquita, B. Emotions in collectivist and individualist contexts. J. Pers. Soc. Psychol. 2001, 80, 68-74. [CrossRef] [PubMed]

93. Imada, T.; Ellsworth, P.C. Proud Americans and lucky Japanese: Cultural differences in appraisal and corresponding emotion. Emotion 2011, 11, 329-345. [CrossRef] [PubMed]

94. Chaplin, T.M.; Hong, K.; Bergquist, K.; Sinha, R. Gender differences in response to emotional stress: An assessment across subjective, behavioral, and physiological domains and relations to alcohol craving. Alcohol. Clin. Exp. Res. 2008, 32, 1242-1250. [CrossRef] [PubMed]

(C) 2017 by the authors. Licensee MDPI, Basel, Switzerland. This article is an open access article distributed under the terms and conditions of the Creative Commons Attribution (CC BY) license (http:/ / creativecommons.org/licenses/by/4.0/). 